\title{
On the Electronic Structure of the $\mathrm{UO}_{2}$ Molecule
}

\author{
Laura Gagliardi,*,† Björn O. Roos, ${ }^{\ddagger}$ Per-Åke Malmqvist, $;$ and John M. Dyke ${ }^{\S}$ \\ Dipartimento di Chimica "G. Ciamician”, Via F. Selmi 2, 40126 Bologna, Italy, Department of Theoretical \\ Chemistry, Chemical Center, P.O. Box 124, S-221 00 Lund, Sweden, and Department of Chemistry, Highfield, \\ University of Southampton, Southampton, SO17 1BJ, U.K.
}

Received: July 26, 2001; In Final Form: September 12, 2001

\begin{abstract}
The structure and vibrational frequencies of the $\mathrm{UO}_{2}$ molecule have been determined using multiconfigurational wave functions (CASSCF/CASPT2), together with a newly developed method to treat spin-orbit coupling. The molecule has been found to have a $(5 \mathrm{f} \phi)(7 \mathrm{~s}),{ }^{3} \Phi_{\mathrm{u}}, \Omega=2$ ground state with a U-O bond distance of $1.77 \AA$. The computed antisymmetric stretching $\sigma_{\mathrm{u}}$ frequency is $923 \mathrm{~cm}^{-1}$ with a $16 / 18$ isotope ratio of 1.0525 which compares with the experimental values of $915 \mathrm{~cm}^{-1}$ and 1.0526 , respectively. Calculations of the first adiabatic ionization energy gave the value $6.17 \mathrm{eV}$, which is $0.7 \mathrm{eV}$ larger than the currently accepted experimental result. Reasons for this difference are suggested.
\end{abstract}

\section{Introduction}

The vibrational frequencies of the triatomics $\mathrm{UO}_{2}, \mathrm{UO}_{2}{ }^{+}$, and $\mathrm{UO}_{2}{ }^{-}$have recently been measured in solid neon and argon matrixes using infrared spectroscopy, with laser ablation techniques. ${ }^{1}$ In addition, the ionization energies of a number of $\mathrm{UO}_{x}$ systems have been measured in the gas phase using electron impact mass spectrometry. ${ }^{2}$ Also, theoretical studies utilizing the density functional approach (DFT/B3LYP) have been performed. This information makes the uranium oxides good candidates for testing more advanced quantum chemical methods for heavy-element compounds. We have recently reported equilibrium geometries and vibrational frequencies for a number of $\mathrm{U}(\mathrm{V})$ and $\mathrm{U}(\mathrm{VI})$ triatomic molecules and their positive ions $\mathrm{XUY}(\mathrm{X}=\mathrm{C}, \mathrm{N}, \mathrm{O})$. The computed frequencies were in agreement with experiment provided that extended basis sets were used. ${ }^{3}$ These calculations were performed using multiconfigurational (CAS) SCF theory with dynamic correlation effects added with second-order perturbation theory (CASPT2). Relativistic effective core potentials (ECP) were used, and no spin-orbit effects were included.

Here we extend this study to the neutral $\mathrm{UO}_{2}$ molecule with two open-shell electrons in the $5 \mathrm{f}$ and $7 \mathrm{~s}$ orbitals. However, in the present study, we have performed all-electron calculations, and spin-orbit coupling has been included.

\section{Method and Details of the Calculations}

The study was performed using the complete active space (CAS) SCF method ${ }^{4}$ with dynamic correlation added using multiconfigurational second-order perturbation theory (CASPT2) ${ }^{5-7}$ Scalar relativistic effects were included using a Douglas-Kroll (DK) Hamiltonian. ${ }^{8,9}$ The effects of spin-orbit (SO) coupling were introduced using a newly developed method based on the CASSCF State Interaction method (CASSI). ${ }^{10,11}$ Here, the CASSCF wave function generated for a number of electronic states are allowed to mix under the influence of a spin-orbit Hamiltonian. The method has recently been de-

\footnotetext{
* Corresponding author. E-mail: laura.g@ciam.unibo.it.

† Dipartimento di Chimica "G. Ciamician", Bologna.

$\doteqdot$ Department of Theoretical Chemistry, Lund.

$\S$ University of Southampton.
}

scribed, and we refer to this article for details. ${ }^{12}$ All calculations have been performed with the software MOLCAS-5. ${ }^{13}$

Three sets of calculations have been performed. All used ANO-type basis sets for $\mathrm{U}$ and $\mathrm{O}$. The $\mathrm{U}$ exponents were optimized using the DK Hamiltonian. The primitive set is 24s19p14d11f. ${ }^{14}$ A small basis set (BS1) used this basis set for $\mathrm{U}$ contracted to $9 \mathrm{~s} 8 \mathrm{p} 7 \mathrm{~d} 5 \mathrm{f}$, and this was combined with the ANO-L basis of the MOLCAS library ${ }^{15}$ for oxygen, contracted to $4 \mathrm{~s} 3 \mathrm{p} 2 \mathrm{~d}$. BS1 was used in the first and second set of calculations with the small and large active space (see below). The third set used the large active space and the larger basis set (BS2), where two g-type functions were added to the U set and one f-type function was added to the $\mathrm{O}$ set.

The ground state of $\mathrm{UO}_{2}$ has been suggested to be $(5 \mathrm{f})(7 \mathrm{~s})$ ${ }^{3} \Phi_{\mathrm{u}}{ }^{1}$ and not $(5 \mathrm{f})^{2}$, as might have been intuitively expected by considering some isoelectronic molecules such as, for example, $\mathrm{NpO}_{2}{ }^{+} \cdot{ }^{16,17}$ In light of this, we decided to use as an active space the $5 \mathrm{f}$ and $7 \mathrm{~s}$ orbitals ( 2 electrons in 8 orbitals, 2/8). This minimal active space gives rise to seven singlet and seven triplet ungerade states arising from the $(5 \mathrm{f})(7 \mathrm{~s})$ electronic configuration and 28 singlet and 21 triplet gerade states from the $(5 \mathrm{f})^{2}$ configuration. State average calculations were performed for all these states in the first set of calculations. Since the gerade and ungerade states do not mix under the influence of spinorbit coupling, one spin-orbit Hamiltonian was constructed for the 91 gerade $\mathrm{SO}$ states and one for the 28 ungerade SO states.

In our recent study of the U(V) and U(VI) systems XUY (X, $\mathrm{Y}=\mathrm{C}, \mathrm{N}, \mathrm{O}),{ }^{3}$ we found that it was important to include in the active space the oxygen $2 \mathrm{p}$ orbitals and the corresponding UO antibonding orbitals of $\sigma$ and $\pi$ type. A second larger active space was therefore constructed, which comprised the six oxygen 2 p orbitals and the corresponding $\sigma$ - and $\pi$-type orbitals on U. They will be hybrid orbitals mixing $5 \mathrm{f}, 6 \mathrm{~d}$, and $7 \mathrm{~s}$. In addition, we have included the four $5 \mathrm{f} \delta$ and $5 \mathrm{f} \phi$ orbitals. In total, this yields 14 electrons in 16 orbitals. CASSCF/CASPT2 calculations of this size are not yet possible. Therefore, the active space was reduced to 14 electrons in 14 orbitals (14/14) by computing only the ground state with this active space. The effect of spin-orbit coupling was taken into account using the results obtained with the smaller active space. 


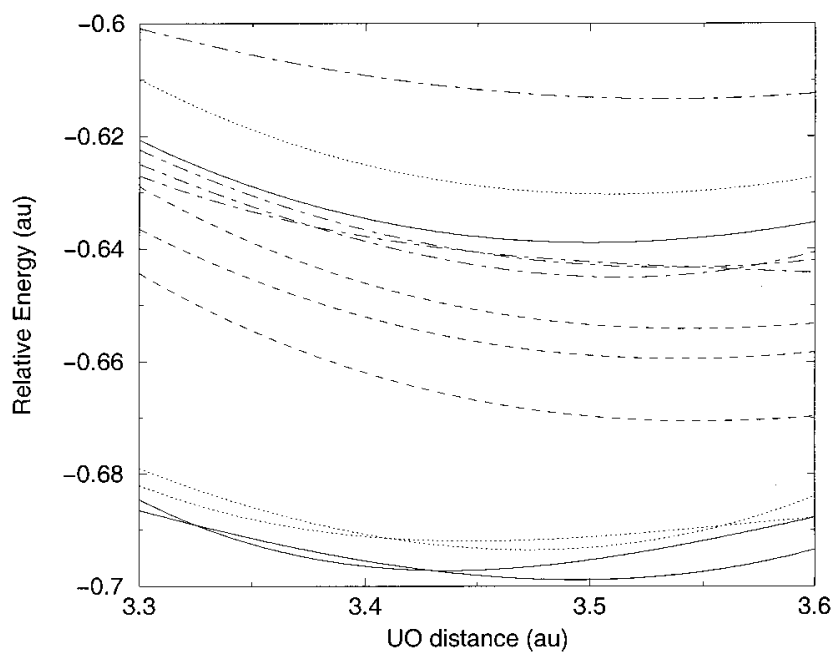

Figure 1. Relative energies (in au) for the lowest electronic states of $\mathrm{UO}_{2}$ as a function of the UO distance at the CASPT2 level of theory (28098 au have been added to the total energies). Solid lines, triplet ungerade states; dotted lines, singlet ungerade states; dashed lines, triplet gerade states; dot-dashed lines, singlet gerade states.

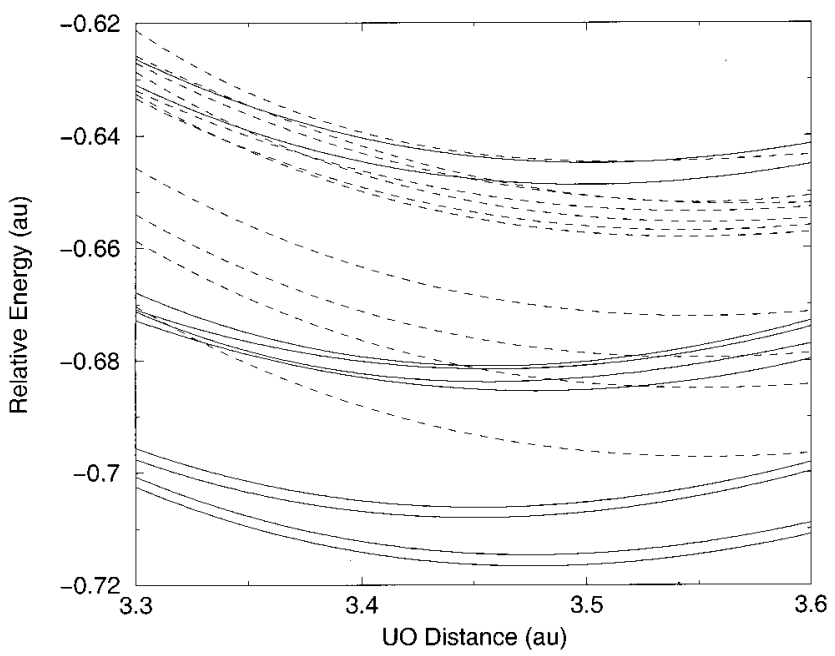

Figure 2. Relative energies (in au) for the lowest electronic states of $\mathrm{UO}_{2}$ as a function of the UO distance at the CASPT2-SO level of theory (28098 au have been added to the total energies). Solid lines, ungerade states; dotted lines, gerade states.

A numerical grid was used to compute the geometry and force constants. The molecule was assumed to have $D_{\infty h}$ symmetry, but only inversion symmetry was used in the geometry optimization in order to allow averaging over all states of a given spin symmetry. Calculations of the asymmetric stretching force constant were made without imposing symmetry. The calculations with the larger active space were, however, performed using symmetry, $D_{2 h}$ or $C_{2 v}$. The bending force constant was not computed, and only linear geometries were studied.

\section{Results}

Figure 1 shows the energies as a function of the UO distance at the CASPT2 level of theory (BS1, 2/8). The four lowest states belong to the (5f)(7s) ungerade configuration, ${ }^{3} \Phi_{\mathrm{u}},{ }^{3} \Delta_{\mathrm{u}},{ }^{1} \Phi_{\mathrm{u}}$, and ${ }^{1} \Delta_{\mathrm{u}}$. The first gerade state is ${ }^{3} \mathrm{H}_{\mathrm{g}}$. Figure 2 shows the effect of spin-orbit coupling. The ungerade states are now strongly mixed resulting in two pairs of almost parallel potential curves. In Table 1, we present the results for the five lowest states, corresponding to $\Omega=2,3,1,2$, and 4 . The results have been
TABLE 1: Analysis of the Spin-Orbit Mixing in the Five Lowest Electronic States of $\mathrm{UO}_{2}\left(\mathrm{BS1}\right.$ and $2 / 8$ active space) ${ }^{a}$

\begin{tabular}{lccccc}
\hline \multicolumn{1}{c}{$\Omega$} & $2_{\mathrm{u}}$ & $3_{\mathrm{u}}$ & $1_{\mathrm{u}}$ & $2_{\mathrm{u}}$ & $4_{\mathrm{g}}$ \\
\hline energy $(\mathrm{eV})$ & 0.00 & 0.05 & 0.24 & 0.29 & 0.52 \\
$r(\mathrm{UO})(\AA):$ & 1.84 & 1.84 & 1.83 & 1.83 & 1.88 \\
force $(\mathrm{au}){ }^{b}$ & 0.86 & 0.85 & 0.83 & 0.83 & 0.87 \\
\hline \multicolumn{7}{c}{ decomposition in $\%$} \\
\hline${ }^{3} \Phi_{\mathrm{u}}$ & 87 & 50 & - & 1 & - \\
${ }^{3} \Delta_{\mathrm{u}}$ & 9 & 15 & 97 & 53 & - \\
${ }^{3} \Pi_{\mathrm{u}}$ & 0 & - & 2 & 3 & - \\
${ }^{1} \Phi_{\mathrm{u}}$ & - & 35 & - & - & - \\
${ }^{1} \Delta_{\mathrm{u}}$ & 4 & - & - & 43 & - \\
${ }^{1} \Pi_{\mathrm{u}}$ & - & - & 1 & - & - \\
${ }^{3} \mathrm{H}_{\mathrm{g}}$ & - & - & - & - & 90 \\
${ }^{3} \Gamma_{\mathrm{g}}$ & - & - & - & - & 2 \\
${ }^{1} \Gamma_{\mathrm{g}}$ & - & - & - & - & 8
\end{tabular}

${ }^{a}$ Relative energies in eV. ${ }^{b}$ Symmetric force constant, $\sigma_{\mathrm{g}}$.

TABLE 2: Relative Energies, Bond Distances, and Symmetric Force Constants for the Lowest Electronic States in the $\mathrm{UO}_{2}$ Molecule $(\mathrm{BS} 1,2 / 8)$

\begin{tabular}{cccc}
\hline method & $\begin{array}{c}T_{\mathrm{e}} \\
(\mathrm{eV})\end{array}$ & $\begin{array}{c}\text { bond distance } \\
(\AA)\end{array}$ & $\begin{array}{c}\text { force } \\
(\mathrm{au})\end{array}$ \\
\hline CASSCF, ${ }^{3} \Phi_{\mathrm{u}}$ & - & 1.75 & 0.75 \\
CASSCF, ${ }^{3} \Delta_{\mathrm{u}}$ & 0.10 & 1.78 & 1.00 \\
CASSCF, ${ }^{3} \mathrm{H}_{\mathrm{g}}$ & 0.03 & 1.86 & 1.28 \\
CASPT2, ${ }^{3} \Phi_{\mathrm{u}}$ & - & 1.84 & 0.72 \\
$\mathrm{CASPT} 2,{ }^{3} \Delta_{\mathrm{u}}$ & 0.04 & 1.83 & 0.97 \\
$\mathrm{CASPT} 2,{ }^{3} \mathrm{H}_{\mathrm{g}}$ & 0.75 & 1.88 & 0.88 \\
$\mathrm{SO}(\Omega=2)_{\mathrm{u}}$ & - & 1.84 & 0.86 \\
$\mathrm{SO}(\Omega=3)_{\mathrm{u}}$ & 0.05 & 1.84 & 0.85 \\
$\mathrm{SO}(\Omega=1)_{\mathrm{u}}$ & 0.24 & 1.83 & 0.83 \\
$\mathrm{SO}(\Omega=2)_{\mathrm{u}}$ & 0.29 & 1.83 & 0.83 \\
$\mathrm{SO}(\Omega=4)_{\mathrm{g}}$ & 0.52 & 1.88 & 0.87
\end{tabular}

obtained with the smaller basis set and active space. This shows how spin-orbit coupling mixes the different states, computed at an UO distance of 3.5 au. The ground state has $\Omega=2$, with extra stabilization due to the interaction of the ${ }^{3} \Phi_{\mathrm{u}}$ state with ${ }^{3} \Delta_{\mathrm{u}}$ and ${ }^{1} \Delta_{\mathrm{u}}$. Only $0.05 \mathrm{eV}$ above is the $\Omega=3$ state, which is a mixture of ${ }^{3} \Phi_{\mathrm{u}},{ }^{3} \Delta_{\mathrm{u}}$, and ${ }^{1} \Phi_{\mathrm{u}}$, still dominated by the ${ }^{3} \Phi_{\mathrm{u}}$ state.

The next state $(\Omega=1)$ is dominated by ${ }^{3} \Delta_{\mathrm{u}}$. The first gerade state $\left({ }^{3} \mathrm{H}_{\mathrm{g}}, \Omega=4\right)$ is found $0.52 \mathrm{eV}$ above the lowest state. We note that the interaction with ${ }^{1} \Gamma_{\mathrm{g}}$ stabilizes the $\Omega=4$ component and makes it the lowest gerade state. In perturbation theory, a first-order spin-orbit term is responsible for the $\Omega=6$ state being the lowest- $\Omega$ gerade state, while a second-order spinorbit term is responsible for the $\Omega=4$ state being the lowest- $\Omega$ gerade state. This second-order effect thus overrules the firstorder spin-orbit effect. Actually the $\Omega=6$ state is found 1.2 $\mathrm{eV}$ above the first gerade state.

The table also gives the relative energies, bond distances, and symmetric force constants. The ungerade states have almost the same bond length and force constant, while the force constant is larger for the first gerade state. More details are given in the Table 2. We notice here that the CASSCF bond lengths are about $0.1 \AA$ too short. The CASPT2 results for the ground state for the bond length and force constant are $1.84 \AA$ and 0.72 au, which are modified to $1.84 \AA$ and 0.86 au by spin-orbit coupling. Thus, the bond length is not affected, but the force constant is because the force constant for the ${ }^{3} \Delta_{\mathrm{u}}$ state is larger than that for the ${ }^{3} \Phi_{\mathrm{u}}$ state.

The vibrational frequencies are presented in Table 3, which also gives the results obtained with the larger 14/14 active space and basis set (BS2). Both these extensions modify the geometry and the force field. As was seen in our earlier studies, ${ }^{3}$ the effect of extending the active space is to make the bond shorter and 
TABLE 3: Bond Distances and Vibrational Frequencies (Intensities within Parentheses) for the Ground State of the $\mathrm{UO}_{2}$ Molecule

\begin{tabular}{|c|c|c|c|c|c|}
\hline method & $r(\mathrm{UO}) \AA$ & $\sigma_{\mathrm{g}}(16)$ & $\sigma_{\mathrm{u}}(16)$ & $\sigma_{\mathrm{u}}(18)$ & $18 / 16$ ratio \\
\hline \multicolumn{6}{|c|}{ Small Basis Set, Small Active Space } \\
\hline CASSCF & 1.845 & 866 & 794(242) & $754(218)$ & 1.053 \\
\hline CASPT2 & 1.845 & 848 & $886(")$ & $841\left(^{\prime \prime}\right)$ & 1.054 \\
\hline $\operatorname{SO}(\Omega=2)$ & 1.841 & 795 & $861\left(^{\prime \prime}\right)$ & $818\left({ }^{\prime \prime}\right)$ & 1.053 \\
\hline $\operatorname{SO}(\Omega=3)$ & 1.841 & 796 & 877(") & 833(") & 1.053 \\
\hline $\operatorname{SO}(\Omega=1)$ & 1.827 & 781 & $855\left(^{\prime \prime}\right)$ & $811(")$ & 1.054 \\
\hline \multicolumn{6}{|c|}{ Small Basis Set, Large Active Space } \\
\hline CASPT2 & 1.831 & 833 & $893(1472)$ & $848(1327)$ & 1.054 \\
\hline $\operatorname{SO}(\Omega=2)$ & 1.786 & 971 & $884(")$ & $839(")$ & 1.054 \\
\hline \multicolumn{6}{|c|}{ Large Basis Set, Large Active Space } \\
\hline CASPT2 & 1.806 & 809 & $932(1485)$ & $885(1339)$ & 1.053 \\
\hline $\mathrm{SO}(\Omega=2)$ & 1.766 & 948 & $923(")$ & $877(")$ & 1.052 \\
\hline \multicolumn{6}{|c|}{ B3LYP-RECP on $\mathrm{U}, 6-311 \mathrm{G}(\mathrm{d}, \mathrm{p})$ on $\mathrm{O}$} \\
\hline & 1.794 & 875 & 937(423) & & \\
\hline \multicolumn{6}{|c|}{$\operatorname{Expt}^{a}$} \\
\hline
\end{tabular}

${ }^{a}$ From ref 1.

stronger. Thus, the bond length decreases (CASPT2) by 0.018 $\AA$. A further decrease of $0.025 \AA$ is obtained by extending the basis set. It is clear that precise determinations of the geometry of these compounds can only be obtained by a careful consideration of both basis sets and active spaces. Spin-orbit coupling also has a considerable effect on the bond length when the larger active space is used. A decrease of $0.045 \AA$ is obtained with BS1, while a decrease of $0.040 \AA$ is obtained with BS2. The final bond length is $1.766 \AA$. It is interesting to compare the CASPT2 bond length, $1.806 \AA$, with our earlier results for $\mathrm{UO}_{2}{ }^{2+}$ and $\mathrm{UO}_{2}{ }^{+} .3$ The results were 1.705 and $1.773 \AA$ obtained with BS2 but without g-type functions on $\mathrm{U}$, which were shown to have only a small effect. Thus, the addition of one $5 \mathrm{f}$ electron on going from the uranyl ion to $\mathrm{UO}_{2}{ }^{+}$increases the bond length by $0.07 \AA$. The further addition of the $7 \mathrm{~s}$ electron gives an additional increase of $0.03 \AA$.

Only the antisymmetric stretching frequency, $\omega\left(\sigma_{\mathrm{u}}\right)$ of $\mathrm{UO}_{2}$ can be compared to the experiment. The neon matrix value reported by Zhou et al. ${ }^{1}$ is $914.8 \mathrm{~cm}^{-1}$ for ${ }^{16} \mathrm{O}$ and $869.2 \mathrm{~cm}^{-1}$ for ${ }^{18} \mathrm{O}$, with a $16 / 18$ ratio of 1.0526 . The best present results give 923,877 , and $1.0525 \mathrm{~cm}^{-1}$, respectively. The matrix effect is estimated to be about $10 \mathrm{~cm}^{-1}$. In addition, the present results correspond to harmonic frequencies. The anharmonic correction can, however, be expected to be small for the $\sigma_{\mathrm{u}}$ vibration. Thus, there is agreement between theory and experiment. We note that the smaller basis set and active space underestimate the frequency.

The results of calculations using DFT (B3LYP with relativistic effective core potentials) are also reported in Table 3 . The results are in reasonable agreement with experiment and identical to the results obtained by Zhou et al. ${ }^{1}$ The results are similar to the ab initio values. One should, however, be careful to conclude that the DFT approach can generally be used to study this class of compounds. Our experience with other similar systems is much less optimistic.

Not much experimental information is available for gaseous $\mathrm{UO}_{2}$. In addition to the measurements of the antisymmetric vibrational frequencies reported above, the ionization energies (IE) have also been measured. The first ionization energy of $\mathrm{UO}_{2}$ has been determined by electron impact mass spectrometry on at least two previous occasions. In 1974, Rauh and Ackermann determined a value of $5.4 \pm 0.1 \mathrm{eV},{ }^{18}$ and in 1999 , Capone et $\mathrm{al}^{2}$ reproduced this result using a molecular beam
TABLE 4: Computed Adiabatic Ionization Energy (in eV) for $\mathrm{UO}_{2}$ with Different Basis Sets and Active Spaces

\begin{tabular}{|c|c|c|}
\hline method & $r(\mathrm{UO})^{a} \AA$ & IE \\
\hline $\begin{array}{l}\text { Sn } \\
\text { CASSCF } \\
\text { CASPT2 } \\
\text { CASPT2-SO }\end{array}$ & $\begin{array}{l}/ 8,1 / 7 \text { Acti } \\
1.721 \\
1.807 \\
1.806\end{array}$ & $\begin{array}{l}5.40 \\
6.24 \\
6.25\end{array}$ \\
\hline \multicolumn{3}{|c|}{ Small Basis Set, 14/14, 13/13 Active Spaces } \\
\hline \multicolumn{3}{|c|}{ Large Basis Set, 14/14, 13/13 Active Spaces } \\
\hline \multicolumn{3}{|c|}{$\begin{array}{c}\text { B3LYP-RECP on } \mathrm{U}, 6-311 \mathrm{G}(\mathrm{d}, \mathrm{p}) \text { on } \mathrm{O} \\
1.759\end{array}$} \\
\hline
\end{tabular}

sample. Gibson obtains $5.5 \mathrm{eV}$ in a similar experiment. ${ }^{19}$ In a recent review paper by Hildenbrand, ${ }^{20}$ electron impact ionization energies are compared with ionization energies determined by more precise methods (e.g., photoelectron spectroscopy), and it is concluded that an electron impact ionization energy is expected to agree with the adiabatic ionization energy to within $\pm 0.01 \mathrm{eV}$. Thus, the above value for the first ionization energy of $\mathrm{UO}_{2}$ might be reliable within the quoted error limits and should be taken as the adiabatic value.

In work reported in 1988, $\mathrm{He}(\mathrm{I})$ photoelectron spectroscopy was used to measure the ionization energies, but insufficient intensity was obtained for $\mathrm{UO}_{2}$ in the low ionization energy region $(5-10 \mathrm{eV}) .{ }^{21}$ Assignments were made with the aid of Hartree-Fock-Slater (LCAO-X $\alpha$ ) calculations. A $(5 \mathrm{f})^{2}$ configuration was, however, assumed for the $\mathrm{UO}_{2}$ ground state, which is different from the results obtained in later studies (including the present).

We have computed the first $\mathrm{IE}$ of $\mathrm{UO}_{2}$. The calculations were performed with both basis sets and with both active spaces. For $\mathrm{UO}_{2}{ }^{+}$, the smaller active space comprised the seven f-orbitals with one active electron. The large active space added the six $\mathrm{O}(2 \mathrm{p})$ orbitals and the corresponding antibonding orbitals (13 electrons in 13 orbitals). The geometry of $\mathrm{UO}_{2}{ }^{+}$was determined in order to compute the adiabatic IE. DFT/B3LYP calculations were also performed. The results are presented in Table 4.

The ground state of $\mathrm{UO}_{2}{ }^{+}$is ${ }^{2} \Phi_{\mathrm{u}}, \Omega=5 / 2$ with a computed bond distance of $1.721,1.807,1.806 \AA$ at the CASSCF, CASPT2, and CASPT2-SO levels, respectively. The CASPT2 value is slightly different from the value obtained in our earlier work, $1.783 \AA$, where ECP's were used for the U atom. The computed IP's are 5.4, 6.2, and $6.2 \mathrm{eV}$, respectively. We note that the effect of spin-orbit coupling is small. This is explained by the fact that the ionized electron resides in an orbital dominated by U(7s). Both the ion and the neutral molecule have one $5 \mathrm{f} \phi$ orbital occupied. No spin-orbit coupling was included in the calculations with the large basis set. The value for the IE is $6.2 \mathrm{eV}$ independent of the method and basis set used. Also, the B3LYP calculations give the same result, and this confirms the value obtained by Zhou et al. ${ }^{1}$ of $6.27 \mathrm{eV}$. The difference between this value and the experimental value, $5.4 \mathrm{eV}$, of 0.7 $\mathrm{eV}$ is surprisingly large. With the large active space and both the small and large basis, we obtain a vertical IE of $6.3 \mathrm{eV}$.

To investigate the difference between the experimental and computed adiabatic IE, a number of checks were made. First of all the $\mathrm{UO}_{2}+{ }^{2} \Sigma_{\mathrm{g}}$ state, obtained on $(7 \mathrm{~s})^{-1}$ ionization from the $\mathrm{UO}_{2}$ ground state, was considered, but this was found to lie more than $1 \mathrm{eV}$ above the ${ }^{2} \Phi_{\mathrm{u}}$ state. Second, the possibility that $\mathrm{UO}_{2}{ }^{+}$could be bent in its ground state was investigated. However, bent $\mathrm{UO}_{2}{ }^{+}$was found to be higher in energy than 
linear $\mathrm{UO}_{2}{ }^{+}$. We therefore concluded that there is no obvious reason our computed value of $6.2 \mathrm{eV}$ for the first adiabatic IE should be in error.

The photoelectron spectrum of uranium recorded with $\mathrm{HeI}$ radiation ${ }^{21}$ exhibits contributions from ionization of the ground state as well as excited states populated at the high temperatures (ca. $2200 \mathrm{~K}$ ) used for evaporation. The band intensities are also affected by autoionization processes. This is also likely to be the case for $\mathrm{UO}_{2}$ prepared by high-temperature evaporation and ionized by photon or electron impact in the energy region 6.0$20.0 \mathrm{eV}$. The calculations with the small active space and small basis sets reveal that several excited states of different symmetry lie in the 5.0-6.50 eV energy region. Among the gerade states, two singlets and two triplets are found at ca. $5.6 \mathrm{eV}$, two triplets at ca. $6.01 \mathrm{eV}$ and two singlets at ca. $6.40 \mathrm{eV}$. If these are positioned above the first ionization energy (ca. $6.2 \mathrm{eV}$ ), then autoionization to the ionic ground state can occur.

Inspection of Table 2 shows that there is a ${ }^{3} \mathrm{H}_{\mathrm{g}}$ state of $\mathrm{UO}_{2}$ at $0.75 \mathrm{eV}$ above the ground state at the CASPT2 level, which corresponds to an excitation energy of $0.52 \mathrm{eV}$ to a $\Omega=4, \mathrm{~g}$ state, when SO coupling in included. The main electronic configuration of the outermost orbitals for this state is $5 \mathrm{f}^{2}$. Clearly, the ground state of $\mathrm{UO}_{2}{ }^{+}$is obtained via a $(7 \mathrm{~s})^{-1}$ ionization from the ground state of $\mathrm{UO}_{2}$, and via a $(5 f)^{-1}$ ionization from the $\mathrm{H}$ state. From tabulation of atomic photoionization cross-sections of Yeh and Lindau, ${ }^{22}$ the $7 \mathrm{~s}: 5 \mathrm{f}$ crosssection ratio is expected to be ca. 1:6. Hence, although the population of the excited $\mathrm{H}$ state is expected to be low at the evaporation temperatures used, its enhanced cross-section may give rise to extra signal below the true adiabatic ionization energy in the electron impact ionization efficiency curve. This may be the reason the experimental value for the first ionization energy of $\mathrm{UO}_{2}$ determined by electron impact mass spectrometry is too low by ca. $0.7 \mathrm{eV}$. Also, we note that the excited state has $\Omega=4$ whereas the ground state has $\Omega=2$. The selection rule $\Delta \Omega=0, \pm 1$ would imply that the excited state is metastable in nature with the transition to the ground state formally forbidden.

As well as contributions from the low-lying excited states below the first ionization energy to the electron-impact ionization efficiency curve, there is also the possibility that excited states above the first ionization energy, populated on electron impact, would give rise to distortion of the ionization efficiency curve. Hence, we present these as possible reasons why the electron impact ionization efficiency curve will be distorted and the ionization energy obtained on extrapolation to zero signal could be in error.

Clearly, there is a need for a determination of the first adiabatic ionization energy of $\mathrm{UO}_{2}$ by more accurate methods other than electron impact mass spectrometry, such as photoelectron spectroscopy or Rydberg series extrapolation methods.

The second IE of $\mathrm{UO}_{2}$ has also been determined experimentally, by Cornehl et al., ${ }^{23}$ who reported a value of $15.4(2.6) \mathrm{eV}$. We computed the second IE with the large active space and both the small and large basis by optimizing $\mathrm{UO}_{2}{ }^{2+}$ in its ${ }^{1} \Sigma_{\mathrm{g}}$ ground state at the 12/12 CASPT2 level. This gave an equilibrium distance of $1.715 \AA$ with the small basis and 1.710 with the large basis. (In our earlier work, we obtained 1.705 and $1.698 \AA$ at ECP's/CASPT2 and ECP's/DFT level, respectively.) The value for the second IE is $14.02 \mathrm{eV}$ with the small basis and $14.36 \mathrm{eV}$ with the large basis. (In our earlier work, we obtained 14.41 and 15.25 eV at ECP's/CASPT2 and ECP's/ DFT level, respectively.) CASPT2 in general gives a lower value for the second IE than DFT. However, all these computed values are within the experimental range.

\section{Conclusions}

We have presented results of calculations on the electronic structure and vibrational frequencies of the $\mathrm{UO}_{2}$ molecule. The aim has been to use this molecule as a test of a newly developed method to include spin-orbit coupling into multiconfigurational wave functions. ${ }^{12}$ The method has been found to work well for systems containing transition metals and main group elements, but this is the first test for an actinide compound.

It is clear from the computed vibrational frequencies that the method gives a satisfactory description of the energy surface around the equilibrium geometry. The effect of spin-orbit coupling is large for the symmetric stretching mode but less significant for the measured antisymmetric stretch. The frequency decreases by $10 \mathrm{~cm}^{-1}$ on including spin-orbit interaction. The molecule has a ground state of ungerade symmetry $(\Omega=2)$, which is best described as the electronic configuration $(5 \mathrm{f} \phi)(7 \mathrm{~s})$ with $D_{\infty h}$ symmetry and a bond length of $1.77 \AA$. The corresponding $(\Omega=3)$ state is, however, located only $0.05 \mathrm{eV}$ above. Spin-orbit coupling mixes states of symmetry ${ }^{3} \Phi_{\mathrm{u}},{ }^{1} \Phi_{\mathrm{u}}$, ${ }^{3} \Delta_{\mathrm{u}}$, and ${ }^{1} \Delta_{\mathrm{u}}$. The first gerade state ${ }^{3} \mathrm{H}_{\mathrm{g}}(\Omega=4)$ is located 0.5 $\mathrm{eV}$ above the ground state.

Calculations of the first ionization energy give a value of $6.2 \mathrm{eV}$, which is $0.7 \mathrm{eV}$ above the generally accepted experimental value of $5.4 \mathrm{eV}$. Our results are very consistent, and they agree with the theoretical value previously determined by Zhou et al. ${ }^{1}$ of $6.27 \mathrm{eV}$. We thus have no obvious reason to suspect that the computed value of $6.2 \mathrm{eV}$ is in error. It is suggested that the possible reason for this difference is that a low value for the first ionization energy has been measured in electron impact mass spectrometric experiments, which involve population of neutral excited states under the high-temperature evaporation conditions used to prepare $\mathrm{UO}_{2}$ in the gas phase. At least one of these excited states, $0.52 \mathrm{eV}$ above the ground state, is long-lived, as the transition to the ground state is forbidden. Further experimental measurements will be required to investigate this suggestion. Calculations of the second ionization energy give values in the region $14.02-14.36 \mathrm{eV}$, which is within the error limits of the experimental value (15.4 $\pm 2.6 \mathrm{eV})$

Acknowledgment. This work was partially supported by Ministero dell' Università e della Ricerca Scientifica, (MURST), and the Swedish Natural Science Research council (NFR). The authors thank Professor Lester Andrews (Virginia) for inspiring the project and useful discussions.

\section{References and Notes}

(1) Zhou, M.; Andrews, L.; Ismail, N.; Marsden, C. J. Phys. Chem. A 2000, 104, 5495 .

(2) Capone, F.; Colle, Y.; Hiernaut, J. P.; Ronchi, C. J. Phys. Chem. A 1999, 103, 10899.

(3) Gagliardi, L.; Roos, B. O. Chem. Phys. Lett. 2000, 331, 229.

(4) Roos, B. O. In Adv. in Chem. Phys.; Ab Initio Methods in Quantum Chemistry - II; Lawley, K. P., Ed.; John Wiley \& Sons Ltd.: Chichester, England, 1987; p 399.

(5) Andersson, K.; Malmqvist, P.-Å.; Roos, B. O.; Sadlej, A. J.; Wolinski, K. J. Phys. Chem. 1990, 94, 5483.

(6) Andersson, K.; Malmqvist, P.-A.; Roos, B. O. J. Chem. Phys. 1992, 96, 1218.

(7) Roos, B. O.; Andersson, K.; Fülscher, M. P.; Malmqvist, P.-Å.; Serrano-Andrés, L.; Pierloot, K.; Merchán, M. In Advances in Chemical Physics: New Methods in Computational Quantum Mechanics; Prigogine, I., Rice, S. A., Eds.; John Wiley \& Sons: New York, 1996; Vol. XCIII, pp 219-331.

(8) Douglas, N.; Kroll, N. M. Ann. Phys. 1974, 82, 89. 
(9) Hess, B. Phys. Rev. A 1986, 33, 3742.

(10) Malmqvist, P.-Å. Int. J. Quantum Chem. 1986, 30, 479.

(11) Malmqvist, P. A.; Roos, B. O. Chem. Phys. Lett. 1989, 155, 189

(12) Malmqvist, P.-Å.; Roos, B. O.; Schimmelpfennig, B. Chem. Phys. Lett., in press.

(13) Andersson, K.; Baryz, M.; Bernhardsson, A.; Blomberg, M. R. A.; Boussard, P.; Cooper, D. L.; Fleig, T.; Fülscher, M. P.; Hess, B.; Karlström,

G.; Lindh, R.; Malmqvist, P.; Neogrady, P.; Olsen, J.; Roos, B. O.; Sadlej, A. J.; Schimmelpfennig, B.; Schütz, M.; Seijo, L.; Serrano, L.; Siegbahn, P. E.; Stålring, J.; Thorsteinsson, T.; Veryazov, V.; Wahlgren, U.; Widmark,

P. MOLCAS, Version 5.0; University of Lund: Lund, Sweden, 2000

(14) Wahlgren, U. Private communication, 2000

(15) Widmark, P.-O.; Malmqvist, P.-Å.; Roos, B. O. Theor. Chim. Acto 1990, 77, 291.
(16) Matsika, S.; Pitzer, R. M.; Reed, D. T. J. Phys. Chem. A 2000, 104, 11983.

(17) Matsika, S.; Zhang, Z.; Brozell, S. R.; Blaudeau, J.-P.; Wang, Q.; Pitzer, R. M. J. Phys. Chem. A 2001, 105, 3825.

(18) Rauh, E. G.; Ackerman, R. J. J. Chem. Phys. 1974, 60, 1396.

(19) Gibson, J. K. Radiochim. Acta 1998, 81, 83.

(20) Hildenbrand, D. L. Int. J. Mass Spectrom. 2000, 197, 237.

(21) Allen, G. C.; Baerends, E. J.; Vernooijs, P.; Dyke, J. M.; Ellis, A. M.; Fehér, M.; Morris, A. J. Chem. Phys. 1988, 89, 5363.

(22) Yeh, J. J.; Lindau, I. At. Data Nucl. Data Tables 1985, 32, 1.

(23) Cornehl, H. H.; Heinemann, C.; Marcalo, J.; de Matos, A. P.; Schwarz, H. Angew. Chem., Int. Ed. Engl. 1996, 35, 891. 